\title{
Tratamiento quirúrgico del Tumor de Koënen: expresión podológica de la Esclerosis Tuberosa
}

\author{
Surgical Treatment of Koenen's Tumor: \\ Expression of Tuberous Sclerosis podiatric
}

\section{Manuel Coheña Jiménez ${ }^{1}$, Pedro Montaño Jiménez², Antonio Sanjuan Rodríguez ${ }^{2}$, Jaime García París ${ }^{3}$, María Reina Bueno ${ }^{2}$}

\author{
${ }^{1}$ Doctor por la Universidad de Sevilla. Diplomado en Podología y Enfermeria. Asistente Honorario del \\ Departamento de Podología. Facultad de Enfermería, Fisioterapia y Podología.Universidad de Sevilla, España. \\ cohmanu@yahoo.es \\ ${ }^{2}$ Doctor en Podología por la Universidad de Sevilla. Profesor colaborador del Departamento de Podología. \\ Facultad de Enfermería, Fisioterapia y Podología. Universidad de Sevilla, España.pmj@us.es; asanjuan@us.es; \\ mreina@us.es \\ ${ }^{3}$ Diplomado en Podología. Asistente Honorario del Departamento de Podología. Facultad de Enfermería, \\ Fisioterapia y Podología.Universidad de Sevilla, España.jaimepodologo@hotmail.com
}

Correspondencia:

Manuel Coheña Jiménez

Departamento de Podología. Centro Docente de Fisioterapia y Podología.

C/ Avicena s/n

E-41009 Sevilla

Correo electrónico: cohmanu@yahoo.es

Fecha de recepción: 15 de diciembre de 2013

Fecha de aceptación: 30 de enero de 2014

Los autores declaran no tener ningún tipo de interés económico o comercial.

\section{RESUMEN}

Los Tumores de Koënen son manifestaciones cutáneas de la Esclerosis Tuberosa, que es de afectación sistémica y en ocasiones con localización en el pie. El conocimiento de este tipo de afecciones dermatológicas es muy importante para un tratamiento correcto y por este motivo un enfoque interdisciplinario es esencial para que el diagnóstico sea preciso y precoz. En la Historia Clínica es importante realizar una valoración de los antecedentes familiares, realizar una exploración de la piel y un estudio neurológico. Se presenta un caso con clínica compatible con la presencia de Tumor de Koënen. El diagnostico diferencial es esencial para plantear el tratamiento y conseguir un buen pronóstico y pronta curación.

Conclusiones. Se plantea el tratamiento quirúrgico como técnica de elección pese a la elevada tasa de recidivas que presenta dicho tratamiento. La resección quirúrgica de la lesión permite el diagnóstico anatomopatologico.

Palabras clave: esclerosis tuberosa; tumor de koënen; tratamiento podológico; tratamiento quirúrgico.

\begin{abstract}
The Koenen tumors are cutaneous manifestations of tuberous sclerosis, which is of systemic involvement and sometimes with location in the foot. The knowledge of this type of dermatological is very important for proper treatment and for this reason an interdisciplinary approach is essential for the accurate and early diagnosis. In the clinical history is important to make an assessment of family history, perform a scan of the skin and a neurological study. We report a case with symptoms consistent with the presence of tumor Koenen. The differential diagnosis is essential for planning treatment and get a good outcome and speedy recovery.

Conclusions. Surgical treatment is proposed as a technique of choice despite the high rate of recurrence presents such treatment. Surgical resection of the lesion allows the pathological diagnosis.
\end{abstract}

Key words: tuberous sclerosis; koënen tumor; podiatric treatment; surgical treatment.

Sumario: 1. Introducción. 2. Esclerosis Tuberosa. 3. Tumor de Koënen. 4. Conlusiones. 5. Informe del caso clínico. Bibliografía.

Referencia normalizada: Coheña Jiménez, M., Montaño Jiménez, P., Sanjuan Rodríguez, A., García París, J., Reina Bueno, M. Tratamiento quirúrgico del Tumor de Koënen: expresión podológica de la Esclerosis Tuberosa. Rev. Int. Cienc. Podol. 2014; 8(2): 75-82. 


\section{INTRODUCCIÓN}

Los Tumores de Koënen son unas de las manifestaciones de la Esclerosis Tuberosa y cuyo conocimiento tiene mayor importancia para el tratamiento podológico de dicha patología. El conocimiento de este tipo de afecciones dermatológicas que se presentan asociadas a enfermedades sistémicas es muy importante para un tratamiento correcto. Dado que los tumores de Koënen son un signo clínico de la Esclerosis Tuberosa $^{1}$ vamos a analizar brevemente dicha patología y su tratamiento mediante el informe de un caso clínico. Por este motivo un enfoque interdisciplinario es esencial para que el diagnóstico sea preciso y precoz.

\section{ESCLEROSIS TUBEROSA}

Desde el punto de vista de la Etimología, podemos decir que la Esclerosis Tuberosa proviene de "Epi" (Epilepsia), "log" (Inteligencia disminuida), y "a" (Adenoma) ${ }^{2}$. Ésta patología también es conocida como la Enfermedad de Bourneville, y definida como un síndrome neurocutáneo, multisistémico, autosómico, y dominante que se caracteriza por el desarrollo de múltiples hamartomas ${ }^{3} \mathrm{y}$ con predisposición aumentada al desarrollo de tumores benignos en tejidos de origen neuroectodermico, tales como los de tipo neurológico y cutáneo ${ }^{4}$. Estos hamartomas son una proliferación de células de tejidos maduros cercanos a la zona afectada, que están distribuidos por todo el cuerpo (piel, cerebro, corazón, riñones, hígado, pulmones) ${ }^{5}$. El término Esclerosis Tuberosa fue acuñado en 1880 por Bourneville y, en 1908, Vogt describe la tríada clásica que se caracteriza por deficiencia mental, crisis epilépticas y angiofibromas faciales. Y cuya manifestación en el pie se caracteriza por la presencia de fibromas subungueales o Tumores de koënen. Actualmente, esta tríada completa sólo se presenta aproximadamente en el $29 \%$ de los pacientes. Henricus Maria Joannes Köenen (1983) estableció la relación entre las lesiones de tumores de Köenen y la Esclerosis Tuberosa. La Esclerosis Tuberosa es un trastorno genético, concretamente es una anomalía congénita del desarrollo embrionario, con manifestaciones en distintos órganos como son la piel, el sistema nervioso, el cerebro y los riñones, además de tener una especial predisposición para el desarrollo de tumores, entre ellos los Tumores de Köenen ${ }^{1}$. Las lesiones cutáneas son las más frecuentes en la Esclerosis Tuberosa seguidas de los angiofibromas que pueden aparecer en cualquier parte del cuerpo ${ }^{3}$.

El diagnóstico se basa en una serie de criterios pues ningún criterio clínico aislado es diagnóstico de dicha patología. El diagnóstico es fundamentalmente clínico y generalmente secundario a las manifestaciones cutáneas ${ }^{6,7}$. De ahí la importancia del conocimiento de dicha patología por el colectivo podológico. Las lesiones cutáneas se aprecian en el $90 \%$ de los pacientes de cualquier edad, y los fibromas periungueales tienden a surgir tras la pubertad. Para diagnosticar Esclerosis Tuberosa se deben reunir dos criterios primarios, o bien, uno primario y dos secundarios. Algunos criterios diagnósticos primarios o de "certeza" son los Angiofibromas faciales, Fibromas ungueales, Hamartoma retiniano, Tuberosidades corticales, Nódulos gliales subependimarios, Angiomiolipomas renales, Placa fibrosa de la frente, y Piel shagreen. Y algunos criterios diagnósticos cuando hay un familiar de primer grado afecto son: Astrocitoma de células gigantes, Rabdomioma cardiaco único o imágenes ecocardiográfica, Tuberosidad cortical única y Hamartoma retiniano único.

Como criterios diagnósticos secundarios o de "presunción" están la presencia de Manchas acrómicas, Hamartoma retiniano peripapilar, Fibromas gingivales, Defectos en el esmalte dentario, Angiomiolipoma solitario renal, Riñón poliquístico, Rabdomioma cardíaco único y presencia de lesiones hipomielinizante subcorticales múltiples o calcificaciones corticales. Los fibromas periungueales de origen no traumático están entre los principales criterios de diagnóstico de las lesiones de la Esclerosis Tuberosa $^{8}$. Son tumoraciones benignas que en ocasiones puede sangrar, ser dolorosa y puede deformar la uña. Se suelen desarrollar una vez pasado el periodo de la pubertad, siendo más frecuentes su afectación en las uñas de los pies que de las manos y se presenta con mayor frecuencia en mujeres que en hombres ${ }^{9}$.

En algunos estudios se concluye que los angiofibromas faciales unilaterales y los fibromas 
periungueales probablemente representen una manifestación clínica poco frecuente de la Esclerosis Tuberosa. Se han descrito algunos casos con afectación unilateral de la enfermedad: angiofibromas faciales unilaterales, fibromas blandos en el lado derecho del cuello, parches de shagreen o nevus de tejido conectivo en la región lumbosacra derecha y fibromas periungueales en el pie derecho pero en la mano derecha. Sin presentar ni evidencia de retraso mental, ni antecedentes de convulsiones ${ }^{10}$.

\section{TUMOR DE KOËNEN}

Los tumores de Koenen constituyen uno de los signos mayores de la Esclerosis Tuberosa, presentándose en casi la mitad de los pacientes que la padecen. Se presentan como proliferaciones periungueales que causan trastornos en la uña y cuyo análisis nos va a permitir un mejor entendimiento de la enfermedad. Estas lesiones son patognomónicos y están presentes hasta en el $50 \%$ de los enfermos de Esclerosis Tuberosa. En la Historia Clínica es importante realizar una valoración de los antecedentes familiares, realizar una exploración de la piel y un estudio neurológico. Igualmente, se recomienda la realización de estudios genéticos entre los familiares.

Los Tumores de Koënen son lesiones tumorales de carácter benigno, generalmente asintomáticas, aunque en ocasiones son dolorosas y requieren tratamiento quirúrgico. Son lesiones periungueales de color rosáceo parduzco, pedunculadas con el extremo apuntado e hiperqueratósico y que se desarrolla en el pliegue proximal y el resto de la lámina ungueal. Son de carácter único o presentación múltiple ${ }^{11}$. Se han descrito varias formas de presentación: en formas de dientes de ajo, formas globosas, $\mathrm{Fu}-$ siformes, etc.. y con afectación periungueal y subungueal ${ }^{12}$. Esta tumoración puede afectar a la lámina ungueal produciendo onicolisis, cromoníquia, surcos y crestas longitudinales y queratosis subungueal. Se pueden localizar en manos y pies $^{13}$. De forma más excepcional, puede presentarse la lesión con localización en el talón del pie siendo un microtraumatismo, el factor desencadenante de la lesión y con un crecimiento progresivo de la misma a lo largo del tiempo ${ }^{14}$.
El Tumor de köenen aparece como manifestación clínica de la enfermedad de PringleBourneville, o bien, secundario a un microtraumatismo continuo en el pie. Su localización en la zona periungueal puede provocar anomalías en la lámina ungueal Las presiones del calzado favorecen el crecimiento del tamaño de las lesiones de las uñas, siendo los traumatismos los que desencadenan la formación de estos tumores ${ }^{12}$. No existe una etiopatogenia clara de esta patología, si bien el factor traumático puede influir en el crecimiento de la lesión ${ }^{14,15}$.

Algunos estudios señalan como localización más frecuente de este tipo de tumores los dedos de los pies ${ }^{9}$. Otros autores ${ }^{12}$ afirman que es más común su presencia en el quinto dedo del pie. Un estudio ${ }^{13}$ de 106 sujetos observa poca frecuencia de los tumores de Koënen y su localización indistintamente tanto en manos (60\%) como en pies $(55 \%)$.

Histológicamente, en el Tumor de Koënen se puede observar una zona distal estrecha formada por fibras de colágeno laxo rico en vasos sanguíneos, y la zona proximal formada por haces de colágeno denso con menos capilares ${ }^{16}$. Podemos encontrar a nivel macroscópico, una lesión de color rosáceo con pápulas que emergen del pliegue proximal de la lámina ungueal y con unas dimensiones que oscilan de 1 hasta $5 \mathrm{~mm}$ desde la base hasta la punta. A nivel epidérmico existe hiperqueratosis con acantosis y un aumento del espesor de la capa granular ${ }^{9}$.

Es importante establecer un diagnóstico diferencial del tumor de Koënen con otras patologías, así como conocer la causa de dicha lesión. Diferenciar con Fibroma, Queloide, Tumor fibroso recurrente de la infancia, Dermatofibrosarcoma protuberans, Dedos supranumerarios, cuerno cutáneo o verruga vulgar, Fibroqueratoma periungueal adquirido fibromatosis digital infantil, poroma ecrino, granuloma piogénico. Algunos autores diferencian entre fibroqueratoma digital adquirido ${ }^{17,18}$ secundario a traumatismo o de forma espontanea y el fibroqueratoma hereditario, por Esclerosis tuberosa, y cuya localización es más proximal ${ }^{19}$.

Existen varias alternativas de tratamiento ${ }^{20}$ como el laser de $\mathrm{CO}_{2}$ ó mediante electrocoagulación, crioterapia para este tipo de tumores, siendo el de tipo quirúrgico el más adecuado, definitivo y aconsejable en determinadas oca- 
siones. La técnica quirúrgica se debe realizar cuidadosamente para evitar las recurrencias. Coincidiendo con Frances C. et al. Y dada la capacidad de recidiva tan elevada que existe tras el tratamiento quirúrgico de este tipo de tumores, se recomienda realizar un seguimiento personalizado sobre la evolución de este tipo de patologías ${ }^{2,21}$. El diagnóstico precoz y una resección completa son fundamentales para evitar posteriores recidivas.

\section{CONCLUSIONES}

El Tumor de Koënen es una de las patologías asociadas a la enfermedad sistémica de la Esclerosis Tuberosa. La presentación de este caso clínico pretende contribuir a la escasa literatura sobre manifestaciones de enfermedades sistémicas con manifestación en el pie. La realización de una buena Historia clínica, analizando posible historia familiar previa, junto con una exploración correcta del paciente puede ayudarnos a un correcto diagnostico y tratamiento de la lesión. Pese a la variedad de opciones de tratamiento disponibles, la resección quirúrgica de la lesión se plantea como tratamiento de elección, pese a la elevada tasa de recidiva que existe en este tipo de tumores.

\section{INFORME DEL CASO CLÍNICO}

Se trata de un varón de 43 años. En la anamnesis presenta una lesión con aspecto rojizo con forma de "diente de ajo", "globulosos", con onicocriptosis en el borde medial del primer dedo del pie izquierdo. No presenta signos de supuración. Según refiere el paciente ha sido secundario posiblemente a un traumatismo ocasional. Destaca la presencia de una lesión de diámetro de $3 \times 4 \mathrm{~mm}$. No presenta antecedentes generales ni podológicos de interés. Se realiza una exploración visual descartando posibles "manchas café con leche" que son comunes y que no presenta, tampoco presenta angiofibromas. Se realiza Historia clínica exhaustiva para descartar los antecedentes genéticos de Escleriosis Tuberosa, por presentar clínica compatible con la presencia de tumor de Koënen. No presenta diagnóstico ni antecedentes familiares de Es- clerosis Tuberosa. La técnica realizada para el tratamiento definitivo ha sido mediante una matriceptomía parcial con técnica Winograd.

La anestesia de la zona se realiza mediante un bloqueo anestésico digital (Figura 1). Se diseña con un lápiz dermográfico (Figura 2) la incisión que vamos a realizar, teniendo en cuenta la importancia de respetar los márgenes de seguridad de la lesión. Se realiza isquemia digital para mantener una correcta visión de todas las estructuras anatómicas en las que vamos a intervenir.

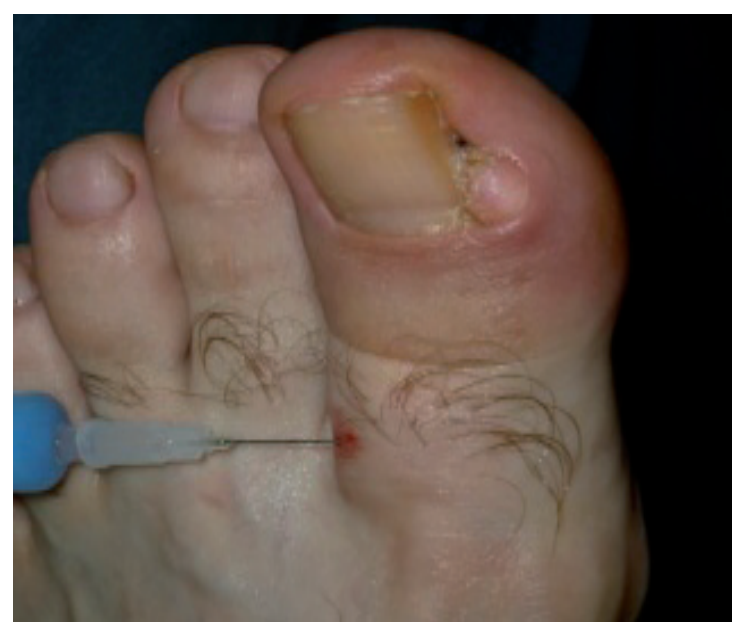

Figura 1. Punción anestésica.

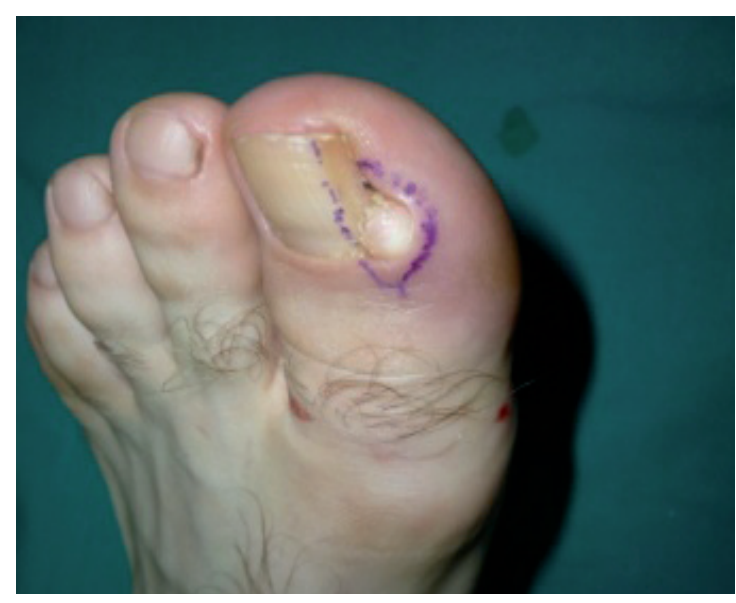

Figura 2. Diseño de incisión.

La secuencia de gestos quirúrgicos comienza con la ayuda de un escoplo (Figura 3) realizamos un aislamiento de la lesión mediante un 
despegamiento ventral de ésta con respecto a la lámina ungueal. También se despega la lámina ungueal del lecho. A continuación, con un alicate inglés cortamos la lámina ungueal (Figura 4). Con un bisturí realizamos el corte de la lesión englobando toda la zona que vamos a extirpar.

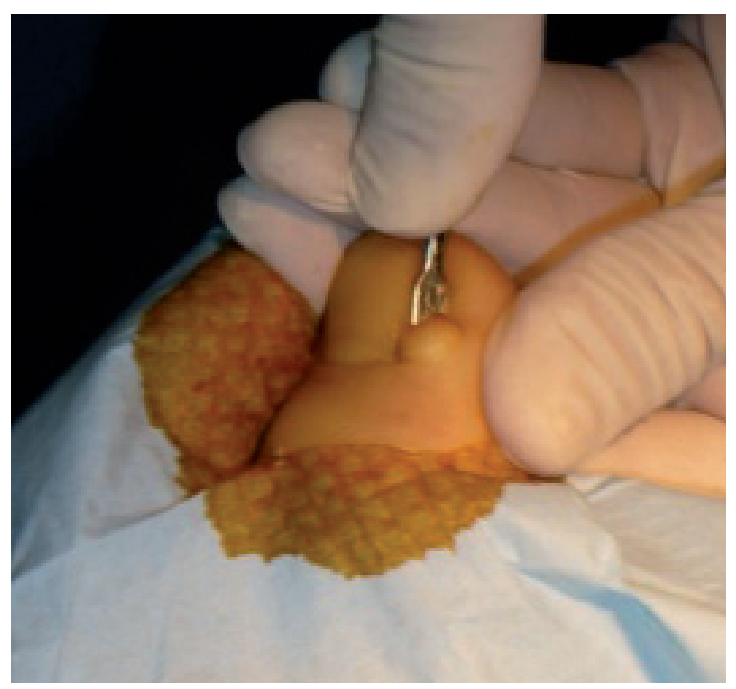

Figura 3. Despegue con escoplo.

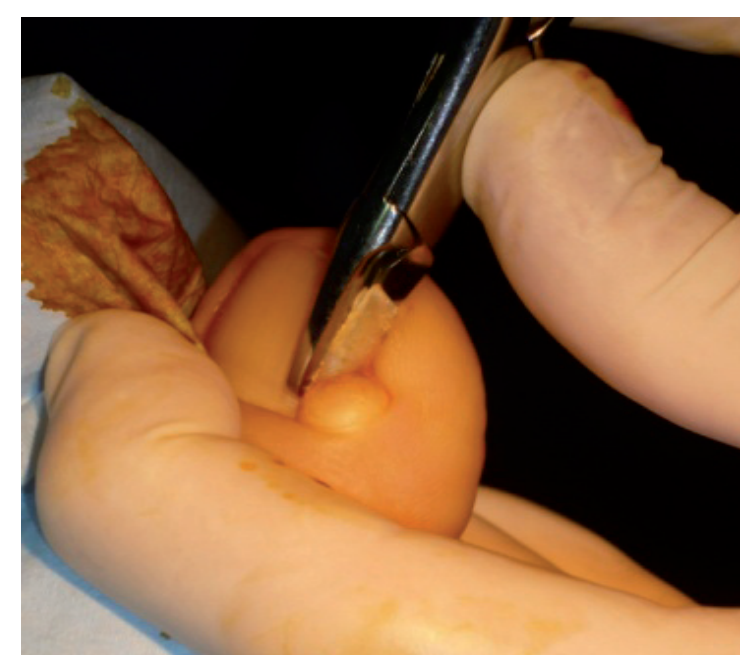

Figura 4. Corte de lámina ungueal.

El corte se inicia desde proximal hacia distal para realizar una incisión semielíptica (Figura 5). Extraemos la porción de uña incluyendo y valorando la zona matricial de ésta (Figura 6).
Completamos la incisión en la parte más medial y englobando toda la lesión.

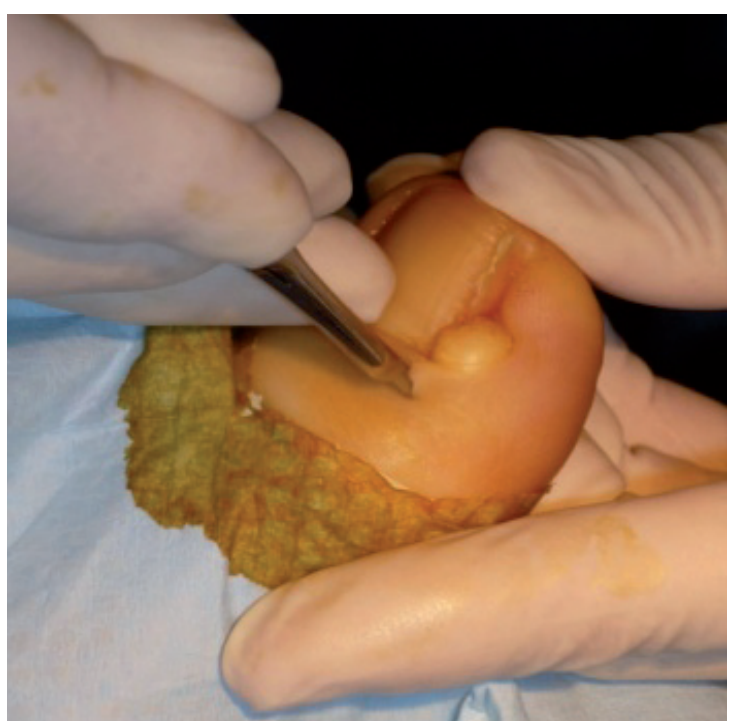

Figura 5. Incisión elíptica.

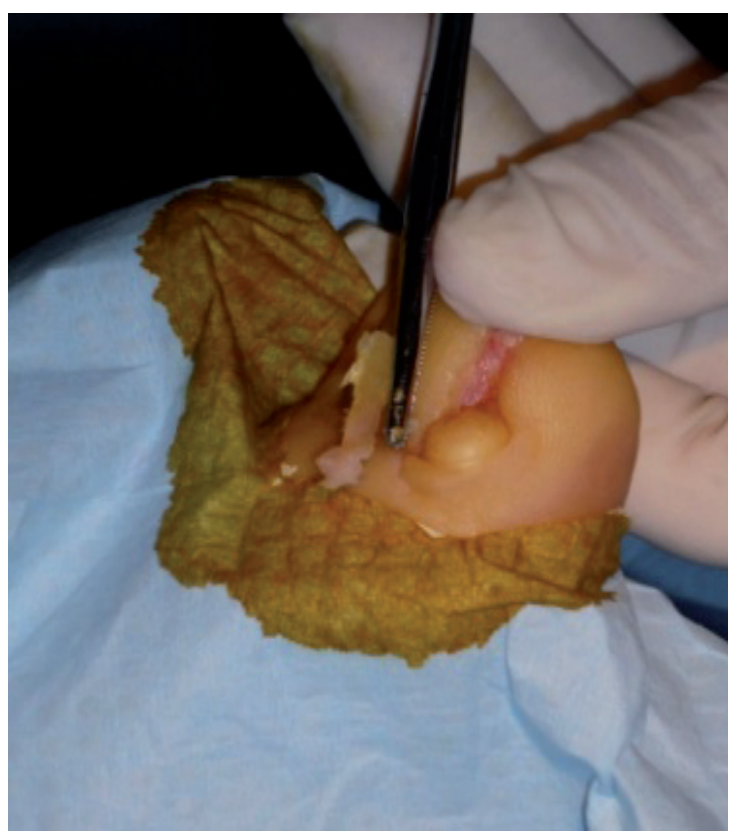

Figura 6. Extracción porción ungueal.

Extirpamos la lesión (Figura 7 y 8) y se introduce en un bote con formol 10\% para su análisis en el laboratorio de anatomía patológica y confirmación diagnóstica (Figura 9). 


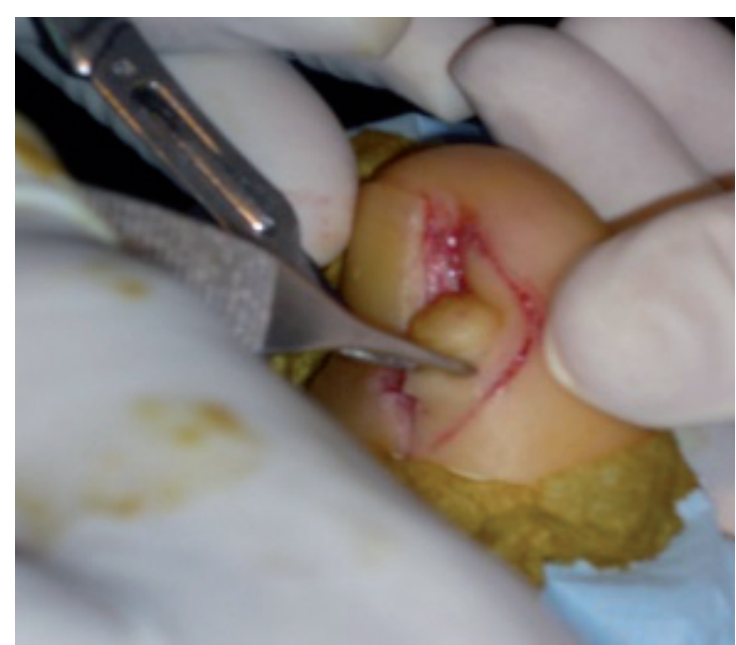

Figura 7. Extirpación de la lesión.

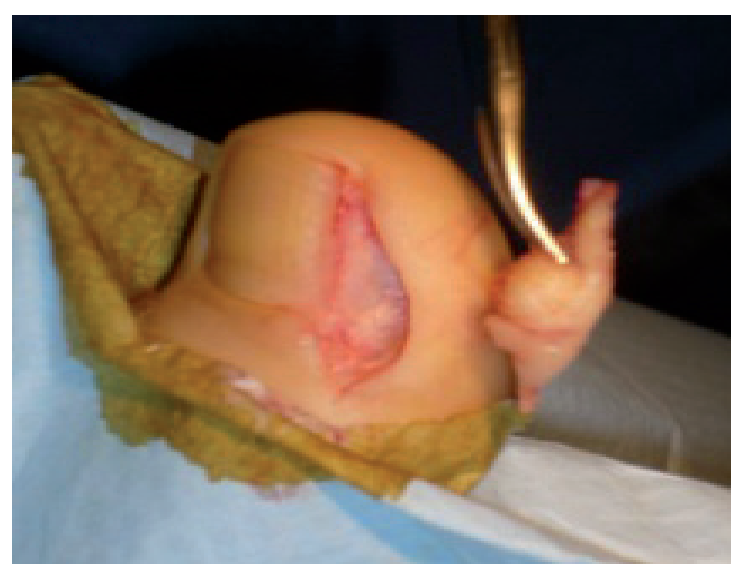

Figura 8. Extirpación de la lesión.

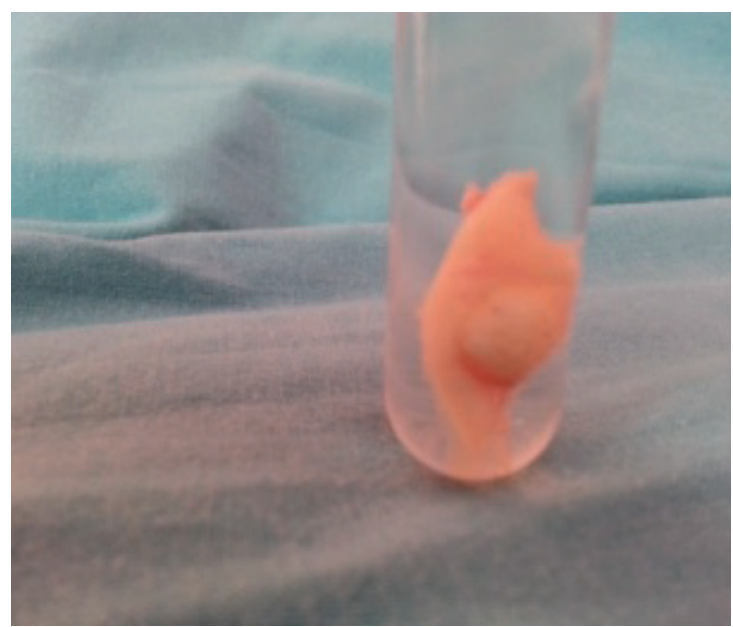

Figura 9. Lesión en Formol.

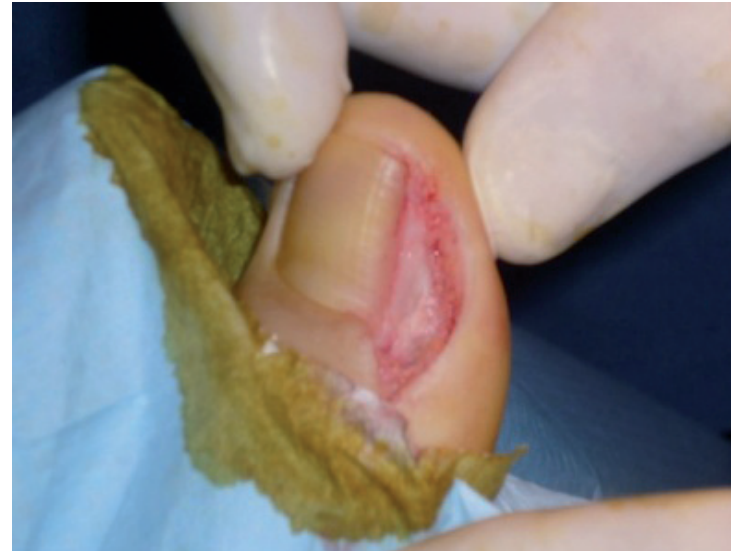

Figura 10. Previa a sutura.

Se coloca sutura con monofilamento no absorbible en la zona más proximal y puntos de aproximación distales, un apósito absorbente y no adherente (Figura 10). Se pauta tratamiento farmacológico antiinflamatorio y analgésico para el postoperatorio inmediato. Se realiza vendaje del dedo con venda cohesiva. Se retira los puntos de sutura a los 12 días y curas secas con una evolución favorable (Figuras 11). Actualmente no se han producido recidivas de la lesión (Figura 12).

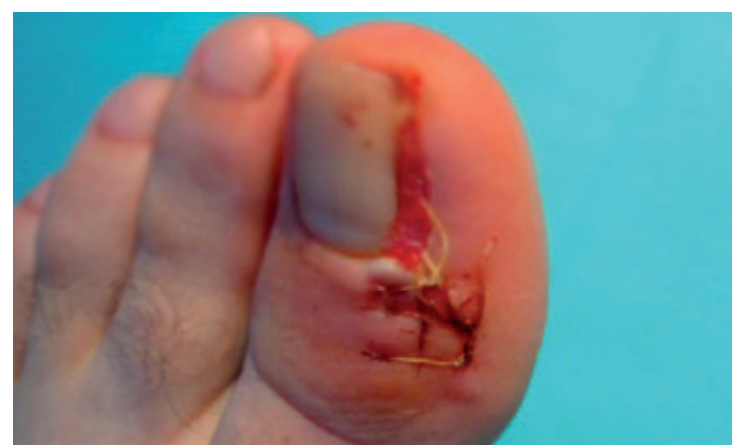

Figura 11. Retirada de suturas.

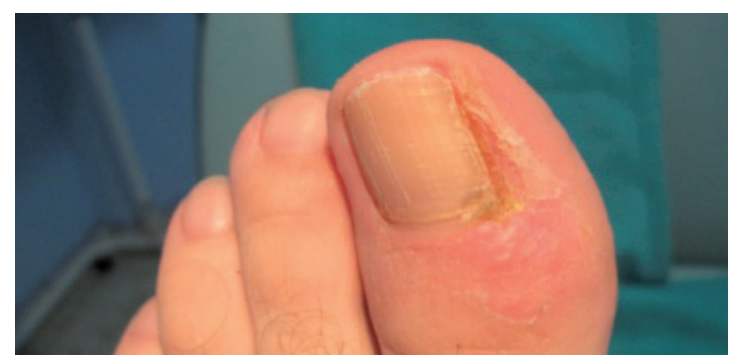

Figura 12. Cura al mes.

Revista Internacional de Ciencias Podológicas 2014, Vol. 8, Núm. 2, 75-82 
En las Figuras 13 y 14 podemos comprobar cómo ha evolucionado el caso mediante la com- probación del aspecto previo de la tumoración y el resultado final tras el tratamiento quirúrgico.

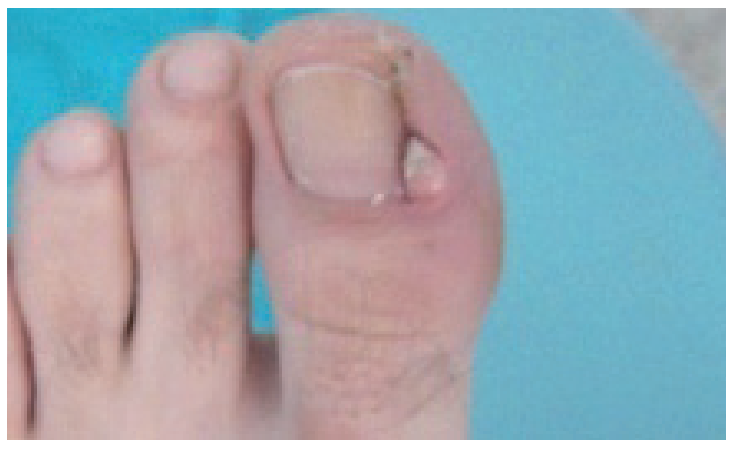

Figura 13. Tumoración previa.

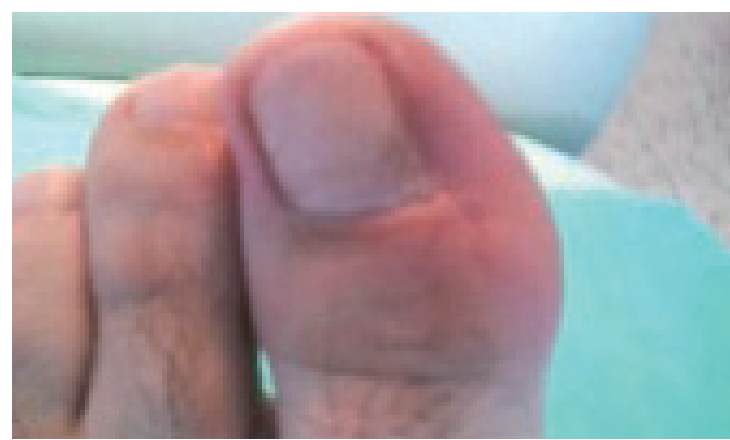

Figura 14. Resultado tras la cirugía.

\section{BIBLIOGRAFÍA}

1. López J, Rodríguez de Rivera E, Márques MS, Finestres F, Chimenos E, y Roselló X. Esclerosis Tuberosa y manifestaciones orales. Caso clínico. Med Oral 2004; 9:216-223.

2. Izquierdo J. Podología Quirúrgica. Cap 6 Otros tumores en el pie. Madrid: Elsevier; 2006: 78-80.

3. Macías M, Sánchez N, Cebollero M, Mandujano G, Velázquez G, et al. Esclerosis tuberosa. Informe de un caso. Rev Esp Patol 2006;39(4):247-249.

4. Valero E, Miñana G y Chorro FJ. Afección cardíaca en la esclerosis tuberosa. Rev Esp Cardiol 2013;66(5):402.

5. Robert A, Fernández G, Kotulska K, et Jozwiak S. Tuberous sclerosis complex: Advances in diagnosis, genetics, and management. J Am Acad Dermatol. New York: Elsevier: 2007;(57):189-202.

6. Crino PB, Nathanson KL et Henske EP. The Tuberous sclerosis complex. N Engl J Med 2006;355: 1345-1356.

7. Hake S. Cutaneous Manifestations of Tuberous Sclerosis. Ochsner J. Fall 2010:3(10):200-204.

8. Quist SR, Ingolf F, Sutter C, Bartram C, Gollnick H, et Leverkus M. Periungual fibroma (Koenen tumors) as isolated sign of tuberous sclerosis complex with tuberous sclerosis complex 1 germline mutation. J Am Acad Dermatol 2010;62(1):159-161.

9. Degin M, Darling T, Moss j, lee C. Histologic variants of periungual fibromas in tuberous sclerosis complex. J Am Acad Dermatol. New York: Elsevier; 2010; (64): 442-444.

10. Segmental tuberous sclerosis in a patient presenting as unilateral facial angiofibromas, periungual fibromas and Shagreen patch. Europ Rew Med Pharmacol Scienc 2011; 15: 980-982.

11. Baran R et Richert B. Common nails tumors. Dermatol clinic 2006; 24(3):297.

12. Aldrich S, Hong C, Groves L, Olsen C, Moss J, et al. Acral Lesions in Tuberous Sclerosis Complex: Insights into Pathogenesis. J Am Acad Dermatol 2010; 63(2): 244-251.

13. Fernández M, Boixeda P, Anaya MJ y Jaén P. Esclerosis Tuberosa. Hallazgos clínicos en 67 pacientes. Act Dermosifiliogr 2009;100:596-601.

14. Martins P, Henrique M, Blatt G, Rochel M, Oliveira L, Peres L, et al. Acquired fibrokeratoma presenting as a giant pedunculated lesion on the heel. Dermatol Online J 2008; 14 (12):10.

15. Zheng L, Huang Y, Han X. Angiofibromas with multiple epidermoid cysts in tuberous sclerosis: new mutation or post-traumatic? J Cutan Pathol 2013;40: 509-512.

16. Villagran A. Tumor de Köenen, presentación de un caso y tratamiento quirúrgico. Rev Scienc Med 2000;21(1):1-2.

17. López D, Cortizas I, Abal C, Barriuso M, Sánchez X y López L. El fibroma periungueal: diagnóstico diferencial. Rev Andal de Podol: Salud del Pie 2004;33:6-9.

18. López D, López L, Barriuso M, Alvarez Ó y Fornos B. Fibroqueratoma periungueal adquirido: tratamiento quirúrgico. Rev Intern Ciencias Podol 2007; 1(2):47-53. 
19. Kakurai M, Yamada T, Kiyosawa T, Ohtsuki M, et Nakagawa H. Giant acquired digital fibrokeratoma. J Am Acad Dermatol 2003; 48(3):67-68.

20. Berlin AL, Billick RC. Use of $\mathrm{CO} 2$ Laser in the treatment of periungueal fibromas associated with Tuberous Sclerosis. Dermatol Surg 2002; 28(1):434.

21. Frances C. Fibromixoma acral superficial, un tumor periungueal CD34 positivo. Actas Dermosifiliogr. 2012; 103 (1): 67-81. 